\title{
Letters to the
}

\section{Editor}

\section{Foreign bodies expectorated through the pneumonectomy stump \\ To the Editor:}

The article by Ahmed and colleagues ${ }^{1}$ in the November 2002 issue, "Benign Expectoration of a Surgical Clip Through a Pneumonectomy Stump," was of interest to me. Certainly all of us performing pulmonary surgery, especially lobectomy and pneumonectomy surgery, are concerned regarding the potential for healing of the bronchial stump and possible breakdown with fistula formation. Various possible causes for such concern include the type of suture (such as silk), the length of the bronchial stump, and the closeness of any tumor or acute infectious process to the line of resection. In addition, the vascularity of the remaining bronchus is certainly important in the healing. More recently, the use of staples and surgical clips in the performance of thoracic surgery has become frequent to reduce the time of the surgical procedure, the amount of air leakage, and the prolonged comorbidity.

The article by Ahmed and colleagues ${ }^{1}$ describes a patient with cystic bronchiectasis who had undergone a right upper lobectomy followed by a completion pneumonectomy. The patient several years later had a coughing spell and expectorated a surgical clip without systemic signs. Ahmed and colleagues ${ }^{1}$ noted that they were unaware of any previous report of nonreactive commonly used titanium surgical clips eroding into the bronchial tree.

Shamji and coworkers ${ }^{2}$ reported on surgical staple metalloptysis after apical bullectomy and placement of pericardial buttresses. They cited possible local inflammation as a cause for erosion. All of us performing pulmonary surgery over a long period have had unusual postoperative occurrences develop. I was among the authors of an article in 1974 entitled "Unusual Expectoration," ${ }^{3}$ in which we reported a number of interesting and unusual items coughed up by patients. These included a bullet and shrapnel. One patient after right lung surgery coughed up 11 of
12 titanium surgical clips. This particular patient brought the clips to us on a routine postoperative visit in an envelope and asked what he should do with them. Chest radiography and physical examination demonstrated no adverse effects from expectoration of these metallic clips. Radiography showed that he retained only 1 surgical clip. A letter was sent to the manufacturing company, inquiring as to the incidence of such an occurrence. No response was ever received from the manufacturer.

I appreciate the article, and I also would suggest that when any foreign body is used in thoracic surgery, particularly in the lung, there is a possibility that the patient will expectorate that foreign body and bring it to the attention of the physician. Ahmed and colleagues' article $^{1}$ was not the first such report, as demonstrated by our report of 27 years before.

Raymond A. Dieter, Jr, MD Dupage Medical Group General Surgery Glen Ellyn, IL 60137

\section{References}

1. Ahmed Z, Kaiser LR, Shrager JB. Benign expectoration of a surgical clip through a pneumonectomy stump. J Thorac Cardiovasc Surg. 2002;124:1025-6.

2. Shamji MF, Manjiak DE, Shamji FM, Matzinger FR, Perkins DG. Surgical staple metalloptysis after apical bullectomy: a reaction to bovine pericardium? Ann Thorac Surg. 2002; 74:258-61.

3. Dieter RA Jr, Cornell R, Hasbrouck P. Unusual expectoration. Am Fam Physician. 1974;10:112-6.

doi:10.1016/S0022-5223(03)00686-X

\section{Chronic and adjustable pulmonary artery banding: Reflections on old knowledge To the Editor:}

Leeuwenburgh and colleagues ${ }^{1}$ presented an elegant article on their novel device for pulmonary artery banding. Some interesting characteristics of right ventricular 
"training" to sustain system pressures were reported 18 years ago ${ }^{2,3}$ and may be of special interest for the development of pulmonary artery banding devices. Ideally, the aim of training of a right ventricle to sustain systemic load should prevent any kind of myocardial damage and probably imitate clinical conditions such as development of pulmonary stenosis. To imitate this type of continuous load increase, we developed and studied a novel device. ${ }^{2}$ The results demonstrate that training of the right ventricle follows very delicate characteristics, at least in pigs. $^{2}$ We found that a fast pressure increase of $3.4 \mathrm{~mm} \mathrm{Hg} / \mathrm{d}$ during 3 weeks was associated with a $20 \%$ to $30 \%$ reduction in right ventricular ejection fraction and a $100 \%$ increase in right ventricular end systolic volume. On the other hand, a slow pressure increase of 1.5 to $2.2 \mathrm{~mm}$ $\mathrm{Hg} / \mathrm{d}$ was associated with less significant functional impairment of the right ventricle. Thus pulmonary artery banding may have harmful effects, which might be even more complex in children with heart disease. Therefore open questions should be addressed before the proposed clinical application of this new device, such as the appropriateness of stepwise load increase in comparison to continuous load increase, with special reference to the implications for right ventricular function. It should also be discussed whether the new device includes refined mechanisms that allow for sensitive adjustment of load increase.

H. H. Sievers, $M D$

Department for Cardiac Surgery University of Luebeck Ratzeberger Allee 160 Luebeck D-23538, Germany

\section{References}

1. Leeuwenburgh BP, Schoof, PH, Steendijk P, Baan J, Mooi WJ, Helbing WA. Chronic and adjustable pulmonary artery banding. $J$ Thorac Cardiovasc Surg. 2003;125:231-7.

2. Lange PE, Sievers HH, Nürnberg JH, Engler K, Pilarczyk D, Onnasch DG, et al. A new device for slow progressive narrowing of vessels. Basic Res Cardiol. 1985;80:430-5.

3. Lange PE, Nürnberg JH, Sievers HH, Onnasch DG, Bernhard A, Heintzen PH. Response of the right ventricle to progressive pressure loading in pigs. Basic Res Cardiol. 1985;80:436-44.

doi:10.1016/S0022-5223(03)00795-5

\section{Reply to the Editor:}

We thank Dr Sievers for his important comments. He is right in pointing out that pulmonary artery banding with the aim of training the subpulmonary ventricle is a delicate procedure that should be done with the utmost caution. The ability of the subpulmonary ventricle to adapt to the increased afterload imposed by the pulmonary artery constrictor depends on the age of the patient, baseline ventricular function, and the timing and level of afterload increase. $^{1,2}$

Dr Sievers points out a study by his group in which it was shown that a slow progression of pressure loading $(1.5 \mathrm{~mm}$ $\mathrm{Hg} / \mathrm{d}$ ) results in a lesser increase of right ventricular end-diastolic pressure and minimal changes in ejection fraction and endsystolic volume relative to a fast progression $(3.6 \mathrm{~mm} \mathrm{Hg} / \mathrm{d})$, thus suggesting better preservation of myocardial function. ${ }^{3}$ In addition, these results were supported by the finding that slow pressure loading resulted in a steeper slope of the relationship between maximal pressure and end-systolic volume, thus reflecting improved contractile performance. The reported functional impairment after fast pressure loading in the study of Lange and colleagues ${ }^{3}$ may be related to the simple fact that a load-dependent index of cardiac performance (ejection fraction) was used to quantify myocardial function after chronic right ventricular pressure overload.

In another article, ${ }^{4}$ we have published the hemodynamic results of our banding study. In that article we showed that stroke volume as well as ejection fraction were decreased, whereas compensatory ventricular dilatation (Frank-Starling mechanism) was not observed after chronic right ventricular pressure loading. In the study, right ventricular pressure overload at systemic (aortic) level was produced within a period of 2 weeks. However, according to the slope and intercept of the end-systolic pressure-volume relationship, right ventricular contractile performance was found to be significantly enhanced in these hearts. In addition, we have shown that abnormalities in diastolic function, associated with the (normal) hypertrophic response to pressure loading, may be held responsible for functional impairment of cardiac function after chronic right ventricular pressure loading. ${ }^{5}$

Part of the difference in results between our study and that of Lange and col- leagues ${ }^{3}$ may be explained by the ages at which the pressure overload was applied. In the latter study, young piglets with an average body weight of $22.3 \mathrm{~kg}$ (range $17-32 \mathrm{~kg}$ ) were subjected to slow progressive right ventricular pressure overload. Apart from differences in species, it is likely that these animals, according to their weight, were substantially older (probably several months ${ }^{6}$ ) than the lambs used in our study (age 2-3 weeks, average body weight $6.4 \pm 1.7 \mathrm{~kg}$ ). This difference may have influenced the myocardial response to pressure loading. Finally, whereas the study by Lange and colleagues ${ }^{3}$ compared results of the banding in animals against their own prebanding values (each animal acted as its own control), we compared right ventricular function after 8 weeks of loading against an age-matched control group without banding.

We agree with Dr Sievers that adjustable constrictors should allow small and reproducible adjustments of the level of constriction. Precisely how small the adjustments can be made with our device cannot be inferred from our current results and needs further study.

Boudewijn P. J. Leeuwenburgh, MSc $c^{a, b}$ Paul H. Schoof, $M D^{c}$ Paul Steendijk, $P h D^{b}$ Jan Baan, $P h D^{b}$ Wolter J. Mooi, MD, PhD Willem A. Helbing, $M D^{a, e}$ Department of Pediatrics (Pediatric Cardiology $)^{a}$

Leiden University Medical Center Leiden, The Netherlands

Department of Cardiology (Cardiac Physiology ${\text { Laboratory })^{b}}$

Leiden University Medical Center

Leiden, The Netherlands

Department of CardioThoracic Surgeryc

Leiden University Medical Center

Leiden, The Netherlands

Department of Pathologyd

The Netherlands Cancer Institute Amsterdam, The Netherlands

Department of Pediatrics (Pediatric Cardiology) ${ }^{e}$ Erasmus Medical Center-Sophia Children's Hospital

Rotterdam, The Netherlands

\section{References}

1. Boutin C, Jonas RA, Sanders SP, Wernovsky G, Mone SM, Colan SD. Rapid two-stage arterial switch operation. Acquisition of left ventricular mass after pulmonary artery banding in infants with transpo- 